\title{
Structural and Rotational Dynamics of Carbon Dioxide in 1-alkyl-3-methylimidazolium bis(trifluoromethylsulfonyl)imide Ionic Liquids: The Effect of Chain Length
}

\author{
Chiara H. Giammanco, Steven A. Yamada, Patrick L. Kramer, Amr Tamimi, and \\ Michael D. Fayer* \\ Department of Chemistry \\ Stanford University, Stanford, CA 94305 \\ *Email: fayer@stanford.edu \\ *Phone: (650) 723-4446
}

\section{Supporting Information}
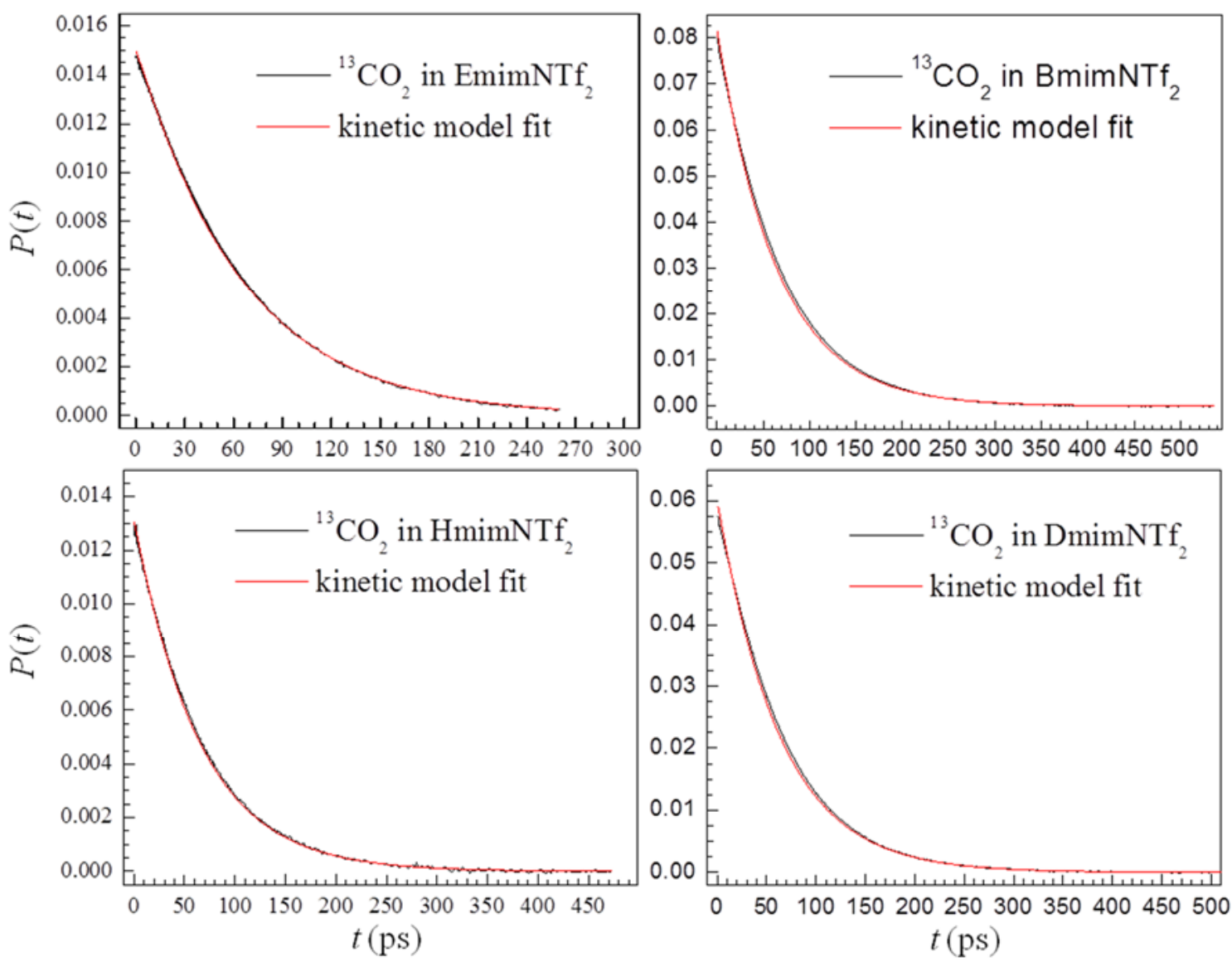

Figure S1. Plots of the vibrational relaxation data at the center of the band (black). The results of the kinetic model fits in EmimNTf $2, B \operatorname{mimNTf} 2, \operatorname{HmimNTf}_{2}$, and DmimNTf 2 are plotted in red. The fitting function includes transfer of population between the main band and the hot bad. The fits give both the asymmetric stretch lifetime and the bend lifetime. 
Table S1. Complete FFCF parameters from the isotropic CLS data.

\begin{tabular}{|c|c|c|c|c|c|c|c|}
\hline Polarization & $\Gamma\left(\mathrm{cm}^{-1}\right)^{*}$ & $\Delta_{1}\left(\mathrm{~cm}^{-1}\right)$ & $\tau_{1}(\mathrm{ps})$ & $\Delta_{2}\left(\mathrm{~cm}^{-1}\right)$ & $\tau_{2}(\mathrm{ps})$ & $\Delta_{3}\left(\mathrm{~cm}^{-1}\right)$ & $\tau_{3}(\mathrm{ps})$ \\
\hline EmimNTf $_{2} \uparrow$ & 3.5 & $0.64 \pm 0.08$ & $2.2 \pm 0.9$ & $0.89 \pm 0.06$ & $14 \pm 4$ & $0.85 \pm 0.09$ & $55 \pm 7$ \\
\hline BmimNTf $_{2}$ & 3.4 & $0.66 \pm 0.05$ & $2.4 \pm 0.6$ & $1.02 \pm 0.03$ & $18 \pm 3$ & $0.93 \pm 0.04$ & $117 \pm 10$ \\
\hline HmimNTf $_{2}$ & 3.2 & $0.82 \pm 0.07$ & $4.9 \pm 0.9$ & $1.13 \pm 0.04$ & $27 \pm 5$ & $1.06 \pm 0.05$ & $290 \pm 50$ \\
\hline DmimNTf $_{2}$ & 3.1 & $0.79 \pm 0.08$ & $5.3 \pm 1.3$ & $1.16 \pm 0.05$ & $31 \pm 7$ & $1.47 \pm 0.06$ & $290 \pm 40$ \\
\hline
\end{tabular}

${ }^{*} \Gamma=$ is the FWHM of the Lorentzian homogeneous linewidth. The frequency fluctuation amplitudes $\Delta_{1}$ and $\Delta_{2}$ are standard deviations of the Gaussian inhomogeneous line shapes.

$\dagger$ These numbers differ with those previously published, ${ }^{39}$ as they have been fit to a triexponential in analogy with the longer chain lengths which require a triexponential rather than a biexponential.

Table S1 contains the FFCF parameters for the generated isotropic data shown in figure 7. The isotropic curves have contributions from structural spectral diffusion and reorientation induced spectral diffusion but do not contain polarization weighting. These are the curves that can be compared to MD simulations. 